\title{
Le syndrome de Lyell
}

Le syndrome de Lyell est une nécrolyse toxique de l'épiderme, le plus souvent d'origine médicamenteuse. Les données les plus récentes semblent indiquer que son mécanisme pourrait être une agression cytotoxique à médiation cellulaire, assez semblable à celle responsable des épidermolyses graves de la réaction du greffon contre l'hôte chez les malades ayant reçu une greffe de moelle.

\section{Jean-Claude Roujeau Assistant d'immunologie et de dermatologie}

\section{ADRESSE ET TIRÉS A PART}

J. C. Roujeau : Service de dermatologie, hôpital 380 e syndrome de Lyell [I, 2] ou nécrolyse toxique de l'épiderme est l'une des formes les plus graves des accidents médicamenteux. Le terme de nécrolyse est un néologisme destiné à combiner les notions de nécrose et de détachement de l'épiderme (épidermolyse).

\section{Un tableau dramatique}

Dans sa forme caractéristique, le syndrome de Lyell réalise en quelques heures un tableau dramatique associant une fièvre élevée, une atteinte érosive de toutes les muqueuses et un érythème cutané diffus sur lequel l'épiderme se détache en vastes lambeaux, laissant à nu un derme rouge-vif suintant ou saignotant ayant un aspect analogue à celui d'une brûlure étendue du $2^{\mathrm{e}}$ degré (figure $I$ ). L'examen histologique montre que l'ensemble de l'épiderme est décollé et nécrotique. L'étendue des décollements, variant de 10 à $100 \%$ de la surface cutanée, est un des facteurs du pronostic. Des manifestations viscérales associées contribuent à la sévérité du tableau. Les plus fréquentes sont les atteintes hépatiques et hématologiques [3] (lymphopénie et granulopénie). Comme chez les brûlés, la destruction de la "barrière épidermique " entraîne des déperditions considérables en eau, en électrolytes, en protides et favorise les infections graves à partir de la flore bactérienne contaminant la peau. Le traitement est purement symptomatique (réhydratation, lutte contre l'infection), aucune thérapeutique étiologique n'ayant fait preuve d'efficacité. La corticothérapie générale est probablement plus nocive qu'utile.

La mortalité du syndrome de Lyell reste considérable $(25 \%$ dans notre expérience). Elle est surtout due aux complications infectieuses. Outre l'étendue des décollements cutanés, le principal facteur de mauvais pronostic est l'âge des patients (nous n'avons déploré qu'un seul décès sur 25 patients de moins de 30 ans contre I 7 chez 42 patients de plus de 50 ans). Chez les survivants, la régénération de l'épiderme est rapide, complète en deux à trois semaines, sans cicatrices autres que des anomalies de la pigmentation, alternance de zones hyperpigmentées et dépigmentées, qui peuvent persister plusieurs mois, voire plusieurs années. La chute des ongles est fréquente, leur repousse habituellement normale. Des séquelles oculaires persistent dans $40 \%$ des cas. Elles sont parfois évolutives et peuvent alors entraîner des lésions cornéennes sévères allant dans quelques cas jusqu'à la cécité. Un syndrome sec* contribue à ces lésions. Il est en tout point sembla- 
ble au syndrome de Sjögren rencontré au carrefour de nombreuses maladies auto-immunes [4]. On retrouve en effet un infiltrat des glandes salivaires par des cellules mononucléées, par contre les anticorps antinucléaires ne sont que rarement positifs (trois cas sur 30, de spécificité anti-Ro/SSA)**.

Il n'y a pas en France de données épidémiologiques sur la fréquence du syndrome de Lyell. La seule étude épidémiologique publiée est déjà ancienne et vient de Suède [5]. Elle montrait pour la région d'Uppsala une incidence de l'ordre de un cas de syndrome de Lyell par million d'habitants et par an. L'expérience des centres français de pharmacovigilance, le recrutement de notre propre service, nous permettent d'aboutir à une estimation voisine. Il doit survenir environ 40 à 50 cas de syndrome de Lyell par an en France.

\section{Les causes}

Tous les participants à une réunion de consensus récemment organisée à Créteil ont convenu que dans l'état actuel des connaissances, les réactions médicamenteuses étaient la seule cause correctement étudiée du syndrome de Lyell. Cependant, environ un tiers des cas restent " idiopathiques", soit par méconnaissance du médicament responsable, soit par intervention d'un autre facteur étiologique.

Certaines souches de Staphylococcus aureus produisent des toxines épidermolytiques (exfoliatines) capables de réaliser chez le nourrisson (mais très exceptionnellement chez l'adulte) un tableau longtemps confondu avec le syndrome de Lyell. Parfois improprement baptisée "Lyell staphylococcique ", cette affection se distingue en fait totalement de la nécrolyse épidermique toxique par l'absence de lésion muqueuse et par un clivage très superficiel, intra-épidermique, donnant une image histologique et un aspect clinique différents [6]. Enfin, si des étiologies infectieuses

\footnotetext{
* Tarissement des sécrétions lacrymales et salivaires.

** V'oir l'article de 7.P. Liautard et coll. dans $\mathrm{m} / \mathrm{s}$ $\mathrm{n}^{\circ} 4$, vol. 1, p. 186 .

$m / s n^{\circ} 7$, vol. 2, septembre 86
}

(notamment herpès récurrent et pneumopathies à Mycoplasma pneumoniae) peuvent induire des syndromes de Stevens-Johnson, cliniquement et histologiquement proches, aucun cas de syndrome de Lyell ne leur a été attribué.

La responsabilité d'un médicament est souvent délicate à établir et l' "imputabilité " repose sur un faisceau d'arguments cliniques essentiellement d'ordre chronologique [7]. On ne dispose actuellement d'aucun test biologique fiable pour confirmer cette responsabilité. La répétition d'accidents analogues avec un même médicament (" imputabilité extrinsèque" des centres de pharmacovigilance) permet de reconnaître quelques médicaments à "haut risque ".

Trois grandes classes de médicaments sont ainsi incriminées dans toutes les publications concernant le syndrome de Lyell. Il s'agit des sulfamides antibactériens, des antiinflammatoires non stéroïdiens et des anti-convulsivants $[2,8]$. Dans le recrutement de notre service $(87$ cas en I I ans avec un médicament incriminé dans 62 cas, voir tableau I, p. suivante), nous avons vu au fil des années se modifier la part respective de ces divers médicaments. Les sulfamides antibactériens étaient il y a quelques années les principaux responsables. L'incidence des cas qui leur ont été attribués est restée à peu près stable. Le principal responsable (du fait de sa diff usion) est le sulfaméthoxazoletriméthoprime qui entraîne environ un cas de syndrome de Lyell pour un million de prescriptions. Au cours des 5 dernières années, les anti-inflammatoires non stéroïdiens ont vu leur part s'accroître et sont devenus les principaux pourvoyeurs de syndrome de Lyell. Si aucun anti-inflammatoire n'est à l'abri de ce type d'accident, deux groupes de médicaments possèdent un risque plus élevé. Il s'agit d'une part de la phénylbutazone et de son dérivé l'oxyphenbutazone et d'autre part des oxicams (piroxicam et, plus récemment et surtout, isoxicam). La part croissante des anti-inflammatoires non stéroïdiens parmi les médicaments inducteurs d'un syndrome de Lyell depuis cinq ans a été surtout liée à cette nouvelle classe d'anti-inflammatoires. Les anticonvulsivants, qui comportent

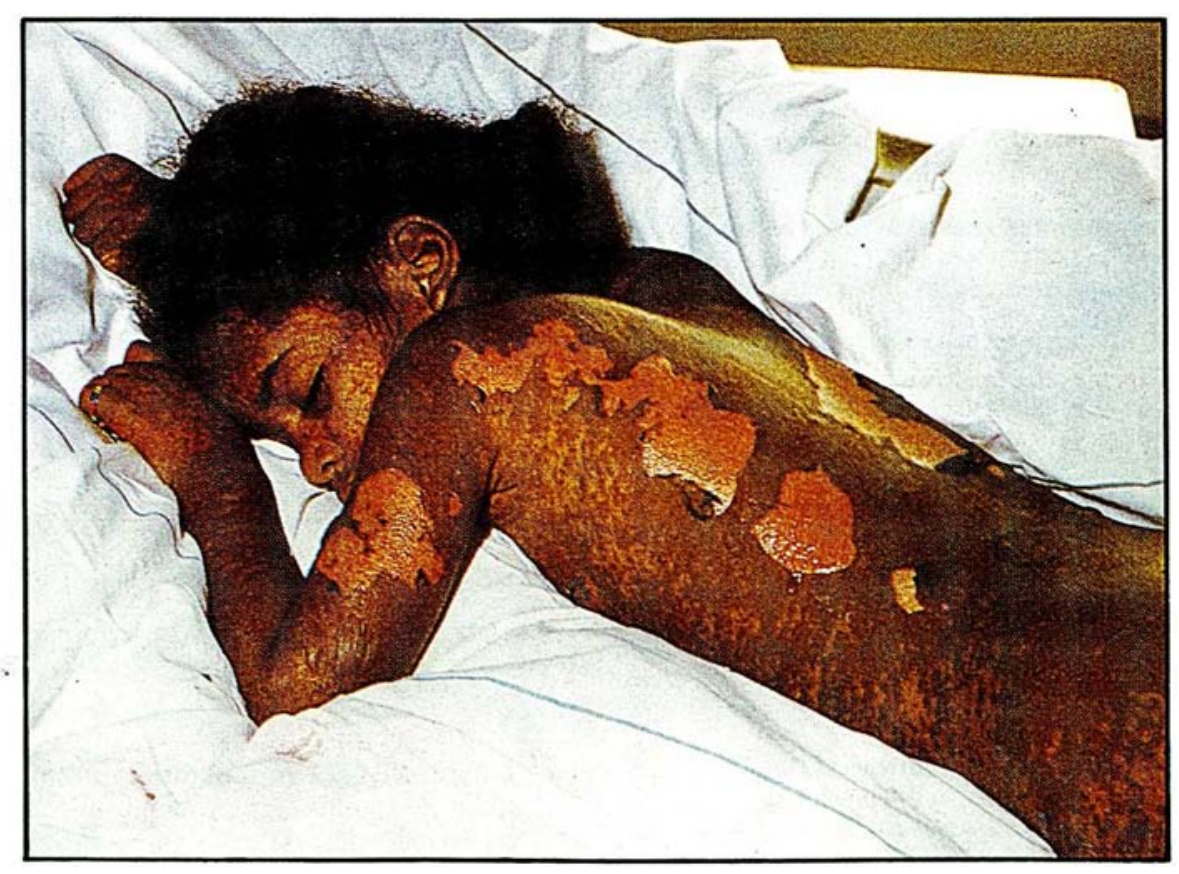

Figure 1. Aspect clinique caractéristique du syndrome de Lyell chez une fillette. 
Tableau I

LES MÉDICAMENTS RESPONSABLES DANS LA SÉRIE DE CRĖTEIL.

(87 patients, 62 observations attribuées à un médicament).

Anti-inflammatoires non stéroïdiens

- oxyphenbutazone (Tanderil')

- Phenylbutazone", Butazolidine

- isoxicam (Vectren ${ }^{\circ}$ )

- piroxicam (Feldène ${ }^{\prime}$ )

- acide niflumique (Nifluril*)

- fenbufène (Cinopal')

- flurbiprofène (Cébutid")

Sulfamides

- sulfaméthoxazole-triméthoprime

(Bactrim, Eusaprim ', Cotrim ${ }^{\circ}$ )

- sulfadiazine (Adiazine)

- salazosulfapyridine (Salazopyrine ${ }^{\circ}$ )

- sulfadoxine (Fansidar ${ }^{\circ}$ )

Anticonvulsivants

- barbituriques

- carbamazépine $\left(\right.$ Tégrétol $^{\circ}$ )

Divers

- allopurinol (Zyloric? Epuric ${ }^{\circ}$ )

- chlormézanone (Supotran ${ }^{\circ}$, Trancopal $^{\circ}$ )

- pyritinol (Encéphabol")
29

11

5

5

5

12

2

2

7

B

I. Lyell A. Toxic epidermal necrolysis: an eruption resembling scalding of the skin. Br 7 Dermatol 1956; 68: 355-6r.

2. Lyell A. Toxic epidermal necrolysis (the scalded skin syndrome): a reappraisal. $\mathrm{Br} 7$ Dermatol 1979; 100: 69-86.

3. Roujeau JC, Moritz S, Guillaume JC, Bombal C, Revuz J, Touraine R. Lymphopenia and abnormal balance of $\mathrm{T}$ lymphocyte subpopulations in toxic epidermal necrolysis. Arch Dermatol Res 1985; 277: 24-7.

4. Roujeau JC, Philippoteau C, Koso M, et al. Sjögren-like syndrome following toxic epidermal necrolysis. Lancet 1985; i: 609-I I.

5. Böttiger LE, Strandberg I, Westerholm B. Drug-induced febrile mucocutaneous syndrome. Acta Med Scand 1975; 198: 229-33

6. Snyder RA, Elias PM. Toxic epidermal necrolysis and staphylococcal scalded skin syndrome. Dermatol Clin I983; r: 235-48.

7. Bégaud B, Evreux JC, Jouglard J, Lagier G. Imputabilité des eff ets inattendus ou toxiques des médicaments. Actualisation de la méthode utilisée en France. Thérapie 1985; 40: I I I-8.

8. Kauppinen $\mathrm{K}$. Cutaneous reactions to drugs with special reference to severe muco-cutaneous bullous eruptions and sulphonamides. Acta Dermatol Venereol 1972; 52: Sup 68.

9. Saurat JH. Cutaneous manif estations of graft versus host disease. Int 7 Dermatol $1981 ; 20$ : 249-56.

Io. Billingham RE, Streilein JW. Toxic epidermal necrolysis and homologous disease in hamsters. Arch Dermatol i968; 98: 528-39.

I . Roujeau JC, Dubertret L, Moritz S, et al. Involvement of macrophages in the pathology of toxic epidermal necrolysis. $\mathrm{Br} 7$ Dermatol 1985;

notamment les barbituriques et la carbamazépine, sont moins fréquemment en cause.

\section{Physiopathologie}

$\mathrm{Si}$ le lien avec des médicaments semble bien établi par des données d'ordre épidémiologique, les mécanismes induisant la nécrose brutale de l'épiderme restent inconnus. Il ne s'agit pas d'un accident de surdosage. On suspecte une réaction d'hypersensibilité, dont les mécanismes restent à démontrer. Les arguments en faveur d'un mécanisme immunologique sont énumérés dans le tableau II ci-dessous.
Dans les cas où le médicament est administré pour la première fois, le syndrome de Lyell survient de façon très caractéristique entre 7 et 2I jours après la première prise du médicament. Cette chronologie est évocatrice d'un mécanisme de sensibilisation immunologique. Dans quelques cas exceptionnels, la reprise du même médicament a pu entraîner des récidives. La récidive a toujours lieu dans les heures suivant la prise médicamenteuse. Cette chronologie évoque une "mémoire " et donc là encore un mécanisme de sensibilisation immunologique. Cette "mémoire" semble spécifique car on n'a pas décrit de

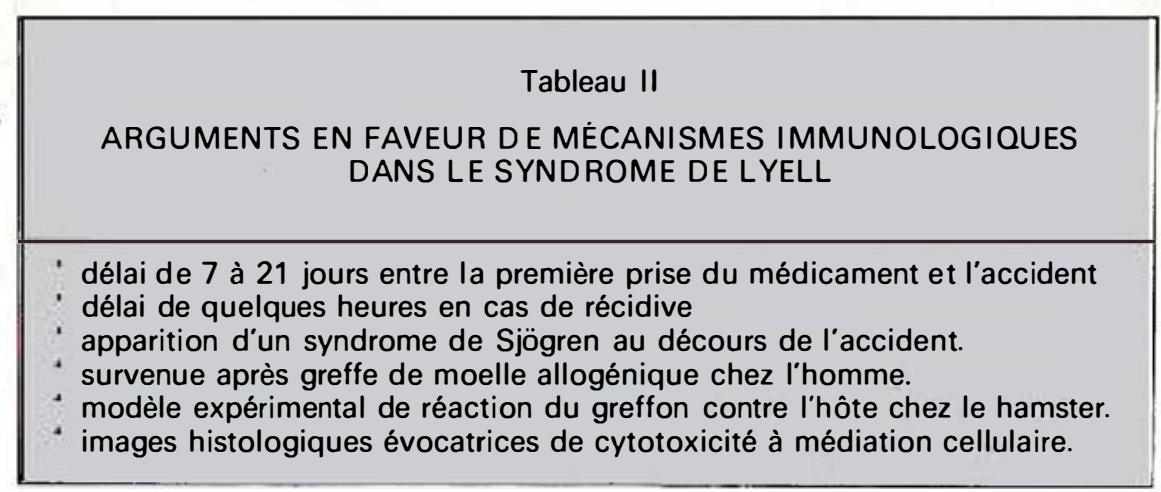




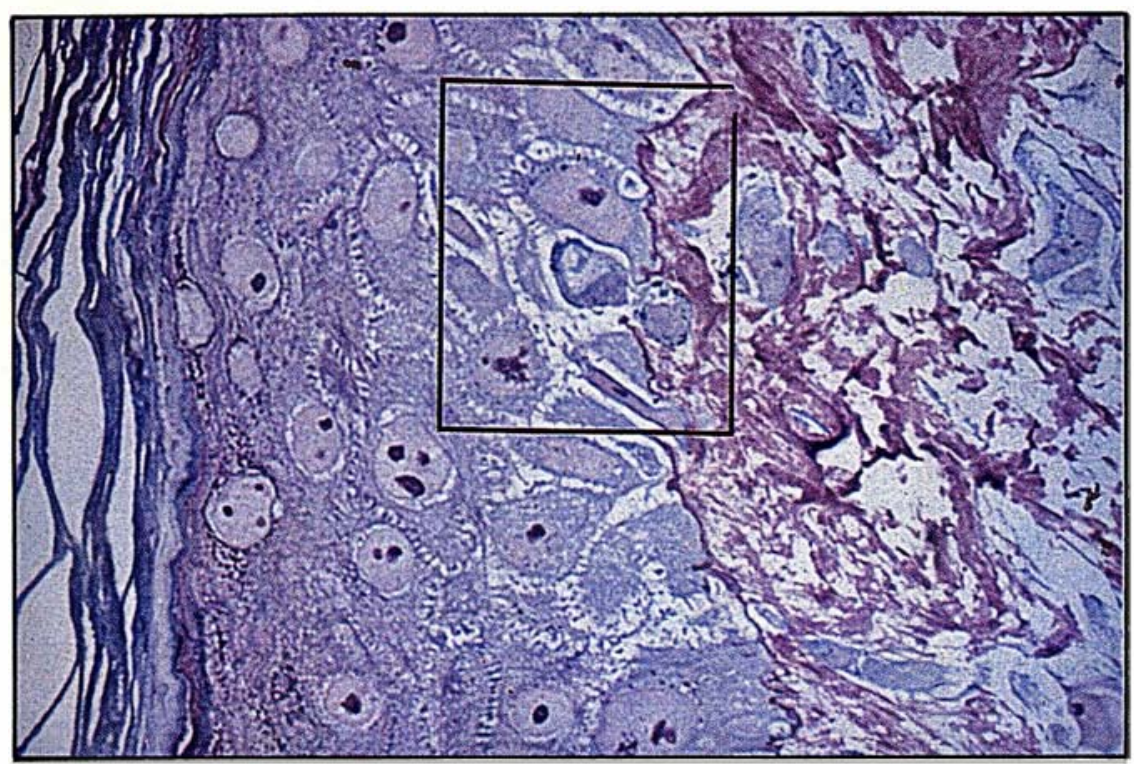

Figure 2. Image histologique très précoce de syndrome de Lyell. L'épiderme (à gauche) est encore pratiquement normal. Dans l'encadré, on reconnaît une cellule mononucléée franchissant la membrane basale, en contact avec un kératinocyte basal en voie de nécrose. La coloration des péroxydases endogènes permet d'affilier la cellule mononucléée au groupe des monocytes macrophages.

récidive avec un médicament différent de celui ayant induit le premier épisode. La " mémoire " est cependant inconstante. La réadministration délibérée du produit suspect effectuée par une équipe finlandaise chez dix patients guéris d'un syndrome de Lyell n'a entraîné qu'une récidive alors que la récidive était quasi constante dans d'autres types d'accidents médicamenteux [8]. Cette "expérience ", éthiquement discutable, montre que l'hypersensibilité à un médicament n'entraîne de syndrome de Lyell que dans des circonstances particulières, chez des patients en état de " réceptivité ".

\section{Le terrain}

Il est troublant de noter que dans nos 29 cas de syndromes de Lyell liés à l'administration d'antiinflammatoires non stéroïdiens, le médicament était le plus souvent prescrit pour des symptômes extraarticulaires fébriles et non pour des rhumatismes chroniques, qui représentent pourtant l'indication de plus de la moitié des ventes de ces produits.

A la phase aiguë du syndrome de $m / s n^{\circ} 7$, vol. 2 , septembre 86
$50 \%$ des patients contre $26 \%$ des témoins, $p<0,0 \mathrm{I})$. Le risque relatif lié à la présence du HLA BI2 (augmentation du risque de survenue de l'accident chez les porteurs de ce phénotype par rapport aux sujets d'autres phénotypes) est faible, calculé à 3,5. Si le lien avec le complexe majeur d'histocompatibilité est faible pour l'ensemble des patients, nous avons eu la surprise d'observer des liens beaucoup plus étroits pour chaque groupe de médicaments responsables. Outre l'association avec Bi2, les cas de syndrome de Lyell aux sulfamides sont associés au phénotype A29, tandis que les cas attribués aux dérivés de l'oxicam sont liés à A2. Le petit nombre de patients dans chaque groupe impose de considérer ces résultats avec prudence en attendant une éventuelle confirmation par d'autres équipes. Un lien avec le complexe majeur d'histocompatibilité a déjà été observé pour d'autres accidents médicamenteux.

\section{Le modèle GVH}

Lyell, nous avons constaté une lymphopénie portant électivement sur les lymphocytes $T_{4}$, alors que la lymphopénie de patients subissant un "stress " intense (polytraumatisés graves) touchait aussi bien les lymphocytes $\mathrm{T} 8$ que $\mathrm{T} 4$. Un tel déficit transitoire en lymphocytes $\mathrm{T}_{4}$ peut être induit par des infections virales. Chez quelques patients présentant un syndrome de Lyell clairement médicamenteux, nous avons observé une séroconversion vis à vis du virus de l'herpès $n^{\circ} I$. Par ailleurs, on connaît la fréquence accrue des réactions cutanées aux médicaments au cours de la mononucléose infectieuse et au cours du SIDA. L'état de "réceptivité " favorisant la survenue d'un syndrome de Lyell pourrait donc être un déséquilibre immunitaire lié à une infection virale aiguë ou chronique.

La recherche d'un terrain prédisposant nous a conduit à étudier les phénotypes HLA des patients ayant présenté un syndrome de Lyell. Dans un groupe de 44 patients, nous avons observé une augmentation significative de l'expression du phénotype HLA BI2 (présent chez
De nombreux cas de syndrome de Lyell ont été observés chez des patients traités par greffe de moelle allogénique [9]. Certains semblent d'origine médicamenteuse, mais la plupart correspondent à des formes extrêmes de réactions aiguës du greffon contre l'hôte (GVH : graft versus host). Ces observations ont d'autant plus d'intérêt pour éclairer la physiopathologie du syndrome de Lyell que le seul modèle expérimental connu de nécrolyse épidermique est précisément une réaction de GVH chez le hamster. Il s'agit d'un modèle complexe impliquant dans un premier temps une réaction de GVH cutanée locale par injection de cellules parentales dans la peau d'un hybride $F_{I}$, puis le développement secondaire d'une nécrolyse non spécifique de l'épiderme [ro]. Étudiée à une époque où l'on ignorait les fonctions immunitaires de l'épiderme, cette nécrolyse expérimentale ne permet pas de comprendre la physiopathologie du syndrome de Lyell. En particulier, ce travail méconnaissait les cellules de Langerhans (cellules dendritiques d'origine médullaire résidant dans l'épiderme, ayant une fonction de pré- 
sentation des antigènes aux lymphocytes et exprimant sur leur membrane les antigènes HLA DR). Ce modèle invite cependant à rechercher des réactions immunopathologiques dirigées contre les cellules épidermiques.

\section{Mécanismes immunologiques}

Quelques auteurs ont constaté des dépôts d'anticorps au site des lésions et la présence d'anticorps anti-épiderme dans le sérum de quelques patients au décours de syndrome de Lyell. Il s'agit en fait de constatations non reproductibles et non spécifiques. Il est fort peu probable que des anticorps participent à la nécrose de l'épiderme. Les lésions épidermiques ne comportent qu'un infiltrat très discret de cellules mononucléées. A partir de lambeaux d'épiderme décollés, nous avons pu caractériser cet infiltrat. Il est composé pour $80 \%$ de phagocytes mononucléés, pour I $5 \%$ de polynucléaires et pour $5 \%$ de lymphocytes, quasi exclusivement T8, phénotype associé aux lymphocytes
T cytotoxiques. Dans des lésions érythémateuses débutantes, nous avons observé des images de contact cellulaire entre une cellule épidermique en voie de nécrose et une cellule mononucléée (figure 2). En matière de $\mathrm{GVH}$, de tels aspects baptisés satellite cell necrosis, sont considérés comme caractéristiques d'une cytotoxicité à médiation cellulaire. Dans le cas du syndrome de Lyell, il s'agissait toujours de phagocytes mononucléés [I I ].

Il est donc tentant de postuler que le syndrome de Lyell résulte d'une réaction cytotoxique contre des structures de membrane des cellules épidermiques modifiées par un médicament ou l'un de ses métabolites. Les gènes du complexe majeur d'histocompatibilité ou une infection virale peuvent, dans cette hypothèse, interférer avec la réponse immunitaire et/ou avec la fixation du médicament aux membranes. La confirmation d'une telle hypothèse nécessite la mise au point de tests in vitro démontrant une interaction entre cellules mononucléées, cellules épidermiques et médicament

\section{Summary}

Lyell syndrome (Toxic epidermal necrolysis: $T E N)$ is the most severe cutaneous adverse drug reaction. The death rate remains very high, around $25 \%$. In our experience most cases were related to drugs, mainly non steroidal anti-inflammatory agents and antibacterial sulfonamides. Several etiological factors are probably associated to account for the very low incidence of the syndrome. The genetic background, linked to the major histocompatibility complex and viral infections may be such factors, enhancing cutaneous hypersensitity to drugs and leading to TEN. The precise ways by which the epidermis is destroyed are unknown. Many indirect pieces of evidence suggest a cell-mediated immune reaction involving the generation of cytotoxic cells against epidermal cells, but this hypothesis must be confirmed by experimental data.

\section{Hommage au Docteur Claude Fortier}

C'est avec une profonde tristesse que nous avons appris le décès de Claude Fortier survenu le 22 avril 1986 à Québec. Claude Fortier était l'un des pionniers de la neuroendocrinologie, science qui consiste en l'étude des relations hormonales entre le cerveau et les glandes endocrines. Elève brillant de Hans Selye, il fut le premier à comprendre l'importance de l'hypothèse émise au lendemain de la seconde guerre mondiale par un chercheur anglais, Geoffrey Harris, selon laquelle le cerveau comme toute glande endocrine, synthétisait des hormones.

Les recherches qu'il a menées dans ce domaine ont abouti à un nombre impressionnant de publications dont certaines font date dans la recherche biomédicale et ont largement contribué à faire $d u$ Québec le point de rencontre de la neuroendocrinologie mondiale. Il a ainsi, au cours des années, regroupé autour de lui de nombreux chercheurs venus $d u$ monde entier pour travailler sur l'axe hypothalamo-hypophyso-surrénalien, et en particulier de France, parmi lesquels Roger Guillemin dont il fut l'ami à l'uni-

Bernard Koch, Fean-Pierre Raynaud. Le développement de la neuroendocrinologie auquel il s'est dévoué sans relâche a permis à deux de ses élèves, Fernand Labrie et Georges Pelletier, de poursuivre l'auvre quil avait entreprise en créant à Québec même un laboratoire de renommée internationale.

Homme de science émérite, humaniste, Claude Fortier m'avait impressionné par sa voix grave, son extraordinaire force de caractère, son aspect sévère qui masquait un profond sens de l'humour. F'aimerais à ce propos, et en hommage amical et respectueux, narrer deux anecdotes. Alors que je travaillais dans son laboratoire depuis un an, il m'avait un jour demandé de participer à l'enseignement à la Faculté de Médecine de l'Université Laval à Québec dont il dirigeait le département de Physiologie. Il me demanda ce qu'en endocrinologie je n'aimerais pas traiter et pour quelles raisons. Après que je lui eus avoué un intérêt limité pour un sujet que je ne dévoilerai pas, il me répondit tout net: "Demain à huit heures, vous exposerez justement ce sujet aux étudiants".
Quelques mois plus tard, il m'expliqua que depuis vingt cing ans, il attendait l'occasion de refaire à quelqu'un le même "coup" que Hans Selye lorsqu'il était arrivé dans son laboratoire.

La seconde anecdote, c'est lui qui me la raconta le jour où il fut nommé Président de la Société Royale du Canada. C'est une farce d'étudiant qui date du temps où, après la guerre, il était chez Hans Selye. Afin d'étudier le stress chez les rats, on avait placé une sirène dans le laboratoire. Une nuit, il mit la sirène en marche à la puissance maximale. Il eut ainsi, mêlé à la foule, l'occasion d'étudier l'effet du stress chez l'homme pendant l'heure qu'il fallut aux pompiers de Montréal pour venir à bout de ce sinistre bruit.

Adieu Docteur Fortier.

William Rostène, Directeur de Recherches Inserm, Ancien élève de Claude Fortier.

* Wade N. La Course au Nobel. Paris: S. Messinger, 198 r. 